\title{
Production of a Discrete, Infectious, Double-stranded DNA by Reverse Transcription in Virions of Moloney Murine Leukemia Virus
}

\author{
D. Baltimore, E. Gilboa, E. Rothenberg, ${ }^{*}$ and F. Yoshimura
}

Department of Biology and Center for Cancer Research, Massachusetts Institute of Technology, Cambridge, Massachusetts 02139

One of the most unique viral replication schemes is that of the retroviruses (a class that includes the RNA tumor viruses). These viruses synthesize a doublestranded (DS) DNA copy of their single-stranded (SS) RNA genome as the initial event following infection of susceptible cells (see Weinberg 1977). The details of this process-called reverse transcription-are still obscure, but the general outlines have become clear during the last few years.

The study of reverse transcription has proceeded along two lines. Some workers have studied nucleic acid molecules extracted from recently infected cells (Weinberg 1977); others have utilized in vitro systems of reverse transcription either with detergent-activated virions or in reconstructed systems (Verma 1977; Taylor 1977). Although the in vitro systems have been useful in studying individual events of the reverse transcription process, only recently have the systems been perfected to the point where they mimic in vivo results.

This paper is concerned with the proof that in vitro systems derived from detergent-activated virions of murine leukemia virus (MuLV) do mimic quite faithfully the events that occur in infected cells. We also describe an unexpected effect of actinomycin D (Act D) on the reverse transcription process and will interpret that effect in the context of a general model of the process of reverse transcription.

\section{In Vivo Reverse Transcription}

The retrovirus genome is a dimer of two $35 \mathrm{~S}$ molecules (Bender et al. 1978), but because the dimeric structure does not enter the reverse transcription process in any known way, we can treat the molecule as if it were a single plus-strand RNA about 9000 nucleotides long.

After a retrovirus infects a cell, the plus-strand RNA is used as a template by the virion-associated reverse transcriptase for synthesis of minus-strand DNA. Synthesis of full-length minus strands requires a few hours, but before completion of the minus strand, plus-strand

*Present address: Memorial Sloan-Kettering Cancer Center, New York, New York 10021.
DNA begins to become evident (Varmus et al. 1978). A major early component of plus-strand DNA is a discrete molecule a few hundred nucleotides long that is initiated somewhere near the $5^{\prime}$ end of the minus-strand DNA and represents a copy of the 5 ' end of the DNA. The final product of reverse transcription includes molecules with full-length minus strands and with partial pieces of plus-strand DNA. Later in infection, closed circular DS DNA becomes evident (for a more complete discussion, see Weinberg [1977]).

\section{Reverse Transcription In Vitro}

Until recently, only the early stages of reverse transcription could be studied in in vitro reactions, because reproducible extensive synthesis of long cDNA could not be achieved (Temin and Baltimore 1972). The early events of reverse transcription, however, have proved fascinating. The primer for minusstrand DNA synthesis has been identified as a specific tRNA hydrogen-bonded to the virion RNA at a position about 100 nucleotides from the $5^{\prime}$ end (Taylor 1977). The early product of reverse transcription is therefore a DNA molecule, called strong-stop DNA, that extends about 100 nucleotides towards the $5^{\prime}$ end of the RNA genome and then stops its synthesis abruptly because it runs out of template (Haseltine et al. 1976). An exact repetition of base sequence at the $5^{\prime}$ end and the $3^{\prime}$ end of the genome allows DNA synthesis to be reinitiated by hybridization of the $3^{\prime}$ end of strong-stop DNA to a position just following the poly(A) of the $3^{\prime}$ end of the viral RNA (see Haseltine and Coffin, this volume). Synthesis of DNA then picks up again, and an extensive copy of the majority of viral RNA is made.

To study events further, it was necessary to optimize the reverse transcription system for the synthesis of minus-strand DNA. In the case of MuLV, it was shown that a combination of a relatively high concentration of deoxyribonucleoside triphosphates (dNTPs), a concentration of magnesium just less than the sum of the triphosphate concentration, and a minimal salt concentration would allow synthesis of the longest possible DNA molecules in reactions that continued over at least 10 hours (Rothenberg and Baltimore 
1976, 1977; Rothenberg et al. 1977). Although the products span a range of sizes, two discrete minusstrand DNAs are made that are approximately the size of the viral RNA. The longer of these two DNA species (8.8-kb DNA) is made in reverse transcription reactions that lack Act $D$. The second, smaller species (8.2-kb DNA) is made both in the presence and in the absence of Act D (Rothenberg et al. 1977).

To understand the effect of Act D, it is important to know whether the 8.2-kb and 8.8-kb DNAs differ from each other at their $3^{\prime}$ ends or their $5^{\prime}$ ends. It has been possible to show that the difference lies at the $3^{\prime}$ end of the molecule (this being the equivalent of the $5^{\prime}$ end of viral RNA). This was demonstrated by forming heteroduplex molecules between the $8.2-\mathrm{kb}$ and $8.8-\mathrm{kb}$ DNAs and the mRNA responsible for the synthesis of the envelope glycoprotein of the MuLV (Rothenberg et al. 1978). This mRNA is a composite, or spliced, molecule, which obtains its leader sequence from the $5^{\prime}$ end of the genome RNA and a majority of its sequence from the $3^{\prime}$ end of the viral genome. It was found that $8.2-\mathrm{kb}$ DNA could hybridize to both parts of the molecule, implying that 8.2-kb DNA extends into the leader sequence. The 8.2-kb DNA, however, did not completely cover the leader sequence. In contrast, the 8.8-kb DNA completely covered the leader sequence and possibly extended beyond it. This result maps the extra 600 bases on $8.8-\mathrm{kb}$ DNA to the $3^{\prime}$ end of the minus-strand DNA molecule and implies that Act $D$ can prevent the addition of the final 600 bases to 8.8 -kb DNA.

To understand reverse transcription, we must then understand why Act $D$ inhibits the synthesis of the longer DNA. Although it is possible that Act D could have a variety of effects, it is a very likely that it acts by inhibiting DNA-dependent DNA synthesis rather than RNA-dependent DNA synthesis (Kahan et al. 1963). Thus, the last 600 nucleotides of $8.8-\mathrm{kb}$ minusstrand DNA would, by this reasoning, result from the copying of DNA rather than RNA. Act D is already known to have one major effect on the reverse transcription process: it prevents synthesis of any plusstrand DNA because plus-strand DNA synthesis is a DNA-dependent process (Manly et al. 1971). An attractive hypothesis is, therefore, that the final 600 nucleotides of $8.8-\mathrm{kb}$ DNA are synthesized as a copy of plus-strand DNA rather than as a copy of viral RNA. We shall return to this point in the final section of this paper.

\section{Synthesis of Infective DNA}

To prove that the "optimal" conditions for reverse transcription can reasonably mimic events in vivo, we have investigated the infectivity of the DNA made in vitro. It was found that after 4 hours of reaction, infective DNA molecules became evident in the reaction mixture, and their concentration increased continually for at least 6 more hours of reaction (Rothenberg et al. 1977). Act D, not surprisingly, prevented the synthesis of infective DNA molecules.
More recently, we have found that the infective DNA molecules are resistant to digestion with the single-strand-specific $S_{1}$ nuclease (Table 1). This implies that the infective DNA represents full-length DS DNA molecules, a result that is consistent with the previous demonstration that the majority of the infectious molecules sediment like full-length DS DNA (Rothenberg et al. 1977).

To examine the DS DNA molecules made during reverse transcription, a preparation of DNA made in vitro was analyzed by neutral agarose gel electrophoresis. A band of DNA of approximately 9000 bp (base pairs) was evident among the products; also evident was a very heterogeneous collection of other molecules (Fig. 1, lane a). Treatment of the reverse transcription product with ribonuclease at either high or low salt helped to clear up the heterogeneous background (Fig. 1, lanes c and e); more importantly, however, treatment of the product with $S_{1}$ nucleasewith or without ribonuclease-resolved a quite discrete band of 9000-bp DNA (Fig. 1, lanes b, d, and f).

When $S_{1}$-nuclease digest products were separated by agarose gel electrophoresis and the individual bands were cut out and tested for their infectivity, it was found that at least $80 \%$ of the infectivity resided in the 9000-bp DNA. Figure 2 shows the DNA that was analyzed and Table 2 demonstrates that the infectivity was found mainly in band B, the 9000-bp DNA. In both Figures 1 and 2, a faint band (A) larger than the 9000-bp DNA is also evident. Preliminary evidence suggests that this is a circularized form of the 9000-bp DNA (E. Gilboa and D. Baltimore, unpubl.).

To study the 9000-bp DNA further, it was compared to the DS DNA made in vivo. This DNA was deteced by the Southern (1975) blotting procedure. In vitro and in vivo products had identical electrophoretic mobilities. Using a series of restriction endonucleases, we compared these products in greater detail. An exact correspondence of bands was observed (Fig. 3), although a few extra bands were found in the in vitro product. (These extra bands appear to have their origin in circular molecules that are presently under investigation.) A map of these fragments based on the analysis of Yoshimura et al. (1979) and Verma and McKennett (1978) is shown in Figure 4. It is evident that both the 5'-end and 3 '-end fragments of the DNA are represented among the restriction fragments that

Table 1. Effect of $S_{1}$-nuclease Digestion on the Infectivity of DNA Synthesized in Purified Virions

\begin{tabular}{ccc}
\hline$S_{1 \text {-nuclease treatment }}$ & DNA $(\mu \mathrm{g})$ & Plaques \\
\hline- & 0.45 & 23 \\
- & 0.90 & 45 \\
+ & 0.45 & 23 \\
+ & 0.90 & 36 \\
\hline
\end{tabular}

DNA was synthesized in purified virions and digested with RNase A. Half of the DNA preparation was subsequently digested with $S_{1}$ nuclease. Infectivity of DNA was tested by transfecting NIH/3T3 cells as described by Rothenberg et al. (1977). 


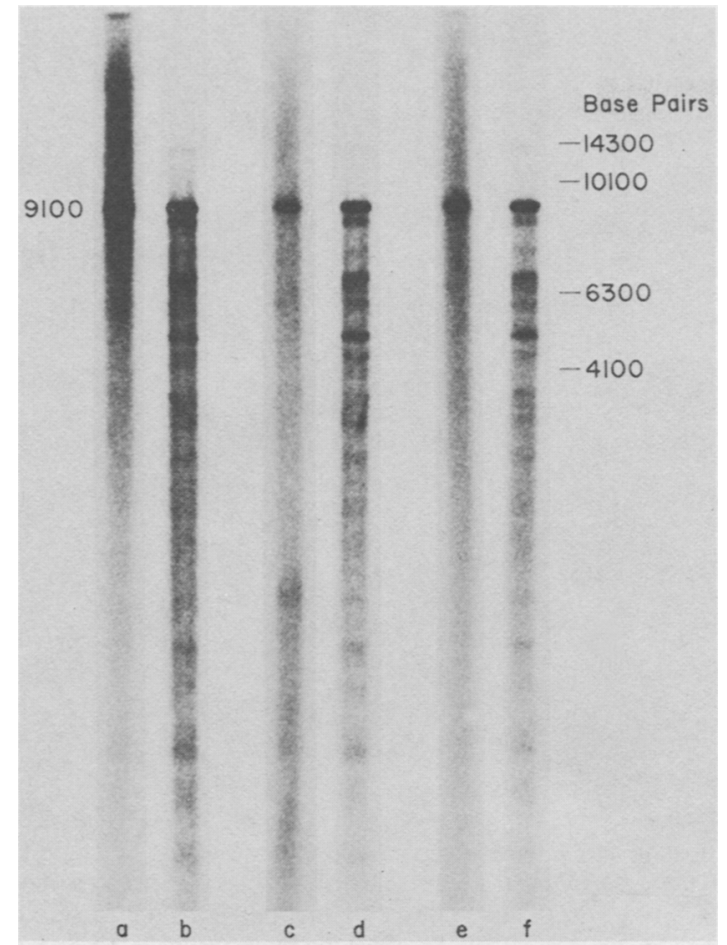

Figure 1. Analysis of DNA synthesized in virions of purified MuLV by electrophoresis through neutral agarose gels. DNA was synthesized in the endogenous reaction as described by Rothenberg et al. (1977) in the presence of $\left[\alpha^{3}{ }^{32} \mathrm{P}\right] \mathrm{dCTP}$. The incubated reaction mixture was digested with $0.2 \mathrm{mg} / \mathrm{ml} \mathrm{pro-}$ teinase $\mathrm{K}$ in the presence of $0.5 \%$ SDS for $30 \mathrm{~min}$ at $37^{\circ} \mathrm{C}$, phenol extracted, precipitated with ethanol, and dissolved in $10 \mathrm{~mm}$ Tris (pH 7.5), and the material excluded by Sephadex G-50 was taken. Portions containing $1.0-1.5 \times 10^{4} \mathrm{cpm}$ were digested with $10 \mu \mathrm{g} / \mathrm{ml} \mathrm{RNase} \mathrm{A} \mathrm{in} 10 \mathrm{mM}$ Tris (pH 7.5), $1 \mathrm{~mm}$ EDTA without (low salt) or with (high salt) $300 \mathrm{~mm}$ $\mathrm{NaCl}$, as indicated, for $60 \mathrm{~min}$ at $37^{\circ} \mathrm{C}$. The products were extracted with phenol and precipitated with ethanol. Subsequent $S_{1}$ nuclease digestion $(+)$ was performed in $30 \mathrm{~mm}$ sodium acetate $(\mathrm{pH} 4.5), 300 \mathrm{mM} \mathrm{NaCl}, 5 \mathrm{mM} \mathrm{ZnCl} 2$ in the presence of $2 \mu \mathrm{g}$ of salmon sperm SS DNA for $60 \mathrm{~min}$ at $45^{\circ} \mathrm{C}$. Samples were extracted with phenol, precipitated with ethanol, dissolved in $40 \mathrm{~mm}$ Tris-acetate (pH 8.3), $1 \mathrm{~mm}$ EDTA, $5 \%$ glycerol and $0.05 \%$ bromphenol blue (BPB), and subjected to electrophoresis in $1 \%$ agarose in $40 \mathrm{mM}$ Trisacetate (pH 8.3), $1 \mathrm{~mm}$ EDTA for $18 \mathrm{hr}$ at $2 \mathrm{~V} / \mathrm{hr}$. Gels were dried and DNA was detected by autoradiography. (a) Untreated DNA; $(b)$ DNA digested with $S_{1}$ nuclease; $(c)$ DNA digested with RNase in the absence of $\mathrm{NaCl}$; $(d)$ DNA digested with RNase in the absence of $\mathrm{NaCl}$ and subsequently digested with $S_{1}$ nuclease; (e) DNA digested with RNase in $300 \mathrm{~mm} \mathrm{NaCl}$; $(f)$ DNA digested with RNase in $300 \mathrm{mM} \mathrm{NaCl}$ and subsequently digested with $S_{1}$ nuclease.

are present in both the in vivo DNA and the in vitro DNA.

To study the SS DNA components of the 9000-bp DNA, it was purified and denatured. Figure 5 compares the pure $9000-\mathrm{bp}$ DNA with a total $S_{1}$ nuclease digest of the reverse transcription product. After denaturation and analysis by electrophoresis through alkaline gels, one band was recovered from the 9000-bp DNA (lane c) that comigrated with the larger $8.8-\mathrm{kb}$ band from the total product (lane d). The major band from the total DNA, the 8.2-kb DNA, was not found in the 9000-bp product.

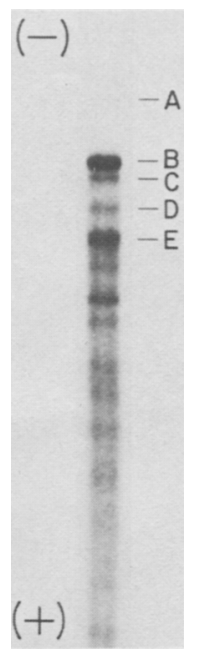

Figure 2. Preparative fractionation of DNA synthesized in purified virions. DNA was synthesized and fractionated on $1 \%$ agarose gels as described in the legend to Fig. 1. DNA from regions $A-E$ was excised and the agarose slice was dissolved in saturated $\mathrm{KI}$ containing $10 \mu \mathrm{g}$ salmon sperm DNA as carrier, passed through $0.5 \mathrm{ml}$ hydroxylapatite at $60^{\circ} \mathrm{C}$, and washed with $10 \mathrm{~mm}$ sodium phosphate $(\mathrm{pH} \mathrm{6.8)}$ and $100 \mathrm{mM} \mathrm{NaCl}$. The bound DNA was eluted with $0.5 \mathrm{M}$ sodium phosphate ( $\mathrm{pH} 6.8$ ), passed through Sephadex G-50, precipitated with ethanol, and used in the experiment described in Table 2 .

These data demonstrate that only the 8.8-kb DNA is present in the 9000-bp DNA. We presume that this 8.8-kb DNA is the minus strand of DNA, although that has yet to be directly demonstrated, and we are uncertain whether the plus strand of DNA is to be found as full-length material or as a broadly heterogeneous collection of molecules that are not evident in Figure 5 (lane c). The 8.2-kb DNA appears to be largely removed by the $S_{1}$-nuclease treatment, implying that most of it contains single-strand regions. It therefore appears likely that the generation of full-length DS DNA is coupled to the synthesis of 8.8-kb DNA and that 8.2-kb DNA can not become converted into fully DS DNA molecules.

\section{Summary and Model}

We have shown that under the appropriate conditions of reverse transcription, detergent-activated vi-

Table 2. Transfection of NIH/3T3 Cells with DNA Fractions Synthesized in Purified Virions

\begin{tabular}{llcc}
\hline \multicolumn{1}{c}{ DNA fraction } & $\begin{array}{c}\text { DNA tested } \\
(\mu \mathrm{g})\end{array}$ & Plaques & $\begin{array}{c}\text { Total initial } \\
\text { infectivity (\%) }\end{array}$ \\
\hline Total S -treated DNA & 0.8 & 17 & \\
Band A & 1.6 & 41 & $(100)$ \\
Band B & 0.024 & 2 & 5 \\
& 0.023 & 7 & \\
Band C & 0.046 & 16 & 81 \\
& 0.011 & 0 & 0 \\
Band D & 0.022 & 0 & 0 \\
Band E & 0.038 & 0 & 0 \\
\hline
\end{tabular}




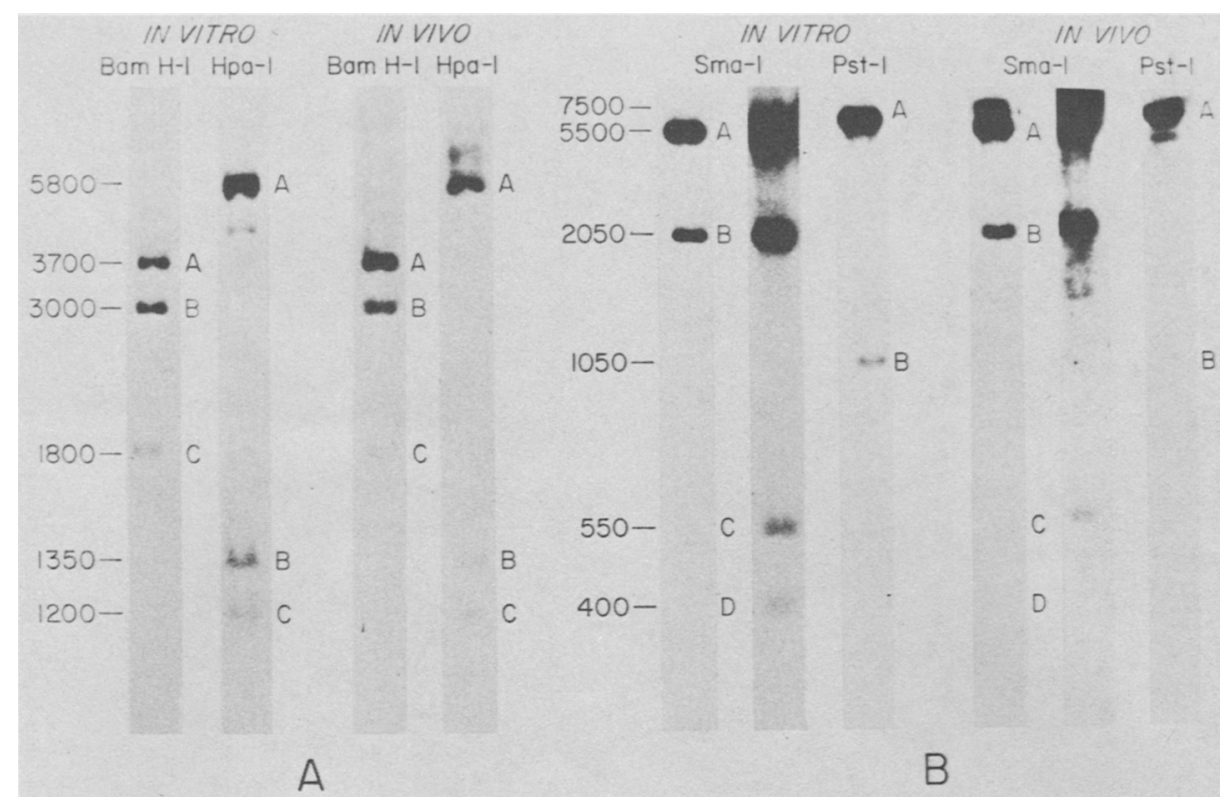

Figure 3. Digestion with restriction enzymes of the in vitro synthesized 9000-bp DNA and the linear form of viral DNA isolated from acutely infected cells. DNA synthesized with $\left[\alpha^{-}{ }^{32}\right.$ p]dCTP in purified virions was prepared as described in the legend to Fig. 1 and the 9000-bp DNA molecule was purified as described in the legend to Fig. 2. The linear form of viral DNA was isolated from acutely infected cells according to the method of Hirt (1967), followed by equilibrium centrifugation in $\mathrm{CsCl}$ to remove circular forms and velocity sedimentation in a sucrose gradient (Gianni et al. 1975). Samples were digested with various restriction enzymes and subjected to electrophoresis on $2 \%$ neutral agarose gels. The digestion products of in vitro synthesized 9000-bp DNA were detected by autoradiography and the restriction fragments derived from the in vivo linear form of viral DNA were detected by the Southern (1975) blotting technique following hybridization with a ${ }^{32}$ P-labeled 35S-virion RNA probe. The numbers indicate the approximate length of the fragments (in $\mathrm{bp}$ ) as estimated from the migration of a SmaI digest of Ad5 DNA (not shown). $A$ and $B$ are two separate gels.

rions will synthesize DS DNA molecules that appear indistinguishable from the linear DS DNA molecules that occur in infected cells. Act $D$ has two effects on this process: it prevents the synthesis of any plusstrand DNA and it prevents the completion of the $3^{\prime}$ end of limit-length minus-strand DNA. There is a model that rationalizes the behavior of this system. The model is derived from the data presented here, from results of earlier experiments performed in our laboratory (Rothenberg et al. 1977, 1978), and from the evidence presented by Taylor et al. and by Varmus et al. (both this volume). Taylor et al. and Varmus et al. have shown that the linear, circular, and integrated forms of viral DNA contain a terminal direct repeat that is approximately the length that we find lacking in DNA made in the presence of Act $D$. We have been able to confirm their results by showing that restriction enzymes that cut the full-length DNA within a few hundred nucleotides from one end also cut the DNA near the other end (E. Gilboa and D. Baltimore, unpubl.). This is consistent with the idea that the last 600 or so nucleotides of the 9000-bp DNA represent a linear extension of the DNA beyond the 5' end of the RNA. The sequences represented by this extension would appear to derive from a recopying of the 3 ' end of the genome (Taylor et al.; Varmus et al.; both this volume).

The model that integrates all of these data is shown in Figure 6. For ease of presentation, we have shown the viral RNA as a circular molecule, but we do not mean to imply that the $3^{\prime}$ end and 5 ' end are physically linked. The first part of the model is similar to that

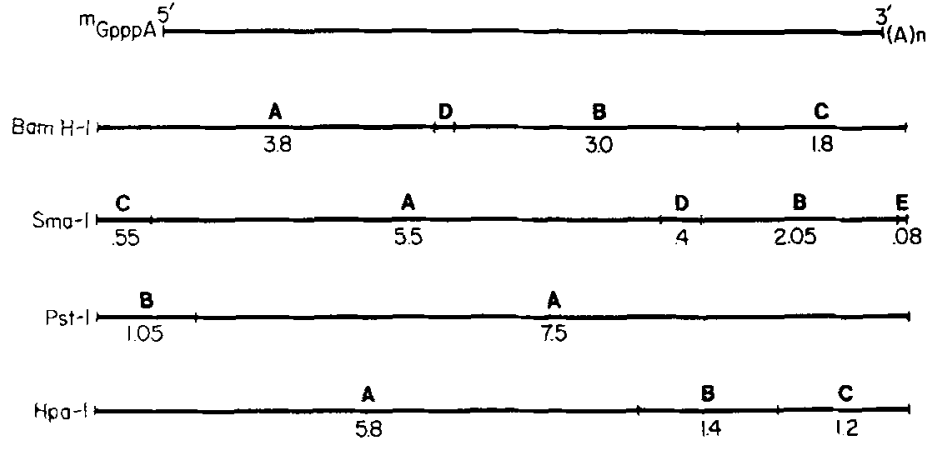

Figure 4. Restriction endonuclease maps of the full-length, linear, unintegrated Moloney murine leukemia proviral DNA. The restriction endonuclease maps were constructed by Yoshimura et al. (1978) for BamHI, SmaI, and PsII and by Verma and McKennett (1978) for BamHI and HpaI. 


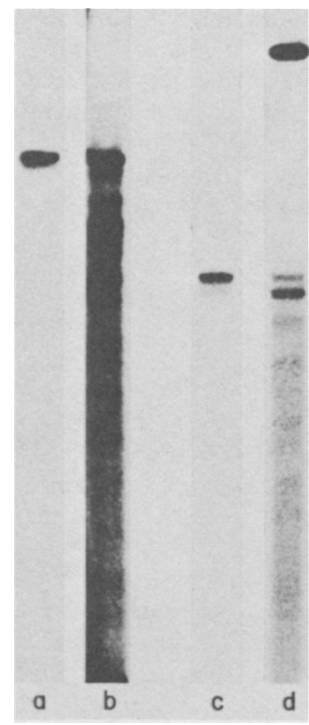

Figure 5. Strand composition of the 9000-bp DNA. DNA was synthesized as described in the legend to Fig. 1 and the $9000-b p$ DNA was isolated as described in the legend to Fig. 2. (a) Purified $9000-b p$ DNA analyzed on a nondenaturing $1 \%$ agarose gel; $(b)$ total unfractionated, $S_{1}$-nuclease-digested DNA analyzed for comparison; (c) purified 9000-bp DNA analyzed under denaturing conditions (30 $\mathrm{mm} \mathrm{NaOH}$ ) in a $1 \%$ agarose gel; $(d)$ total unfractionated DNA analyzed in $30 \mathrm{mM}$ $\mathrm{NaOH}$ for comparison.

presented previously by Haseltine and Baltimore (1976) and Haseltine et al. (this volume) and shows DNA synthesis initiated on a tRNA near the $5^{\prime}$ end of the viral RNA and then jumping to the $3^{\prime}$ end of viral RNA, where synthesis is reinitiated and continues around the RNA. After a certain amount of minusstrand synthesis has occurred, plus-strand synthesis is initiated as implied by the work of Varmus et al. (1978 and this volume). Their work suggests that there is a specific site for initiation, although it is unclear what controls initiation at that site. A piece of plus-strand DNA is then made that extends about 600 nucleotides towards the $3^{\prime}$ end of the RNA. In the presence of Act D, this synthesis does not occur, and the synthesis of minus-strand DNA stops somewhat short of the end of the RNA. What prevents synthesis at that point is obscure.

In the absence of Act D, synthesis is also blocked at that point, but by a mechanism that may be analogous to the original jump that starts minus-strand synthesis, DNA synthesis jumps to the fragment of plusstrand DNA; the final copying of the minus-strand DNA is actually DNA-dependent DNA synthesis. In this way, we account for the Act-D sensitivity of the addition of the final 600 nucleotides to the minus strand. The minus strand generated in this way will have at its 3 ' end an exact repeat of about 600 nucleotides of the sequence at its $5^{\prime}$ end. The sequence homology that enables the minus strand to jump to the plus-strand fragment presumably lies in the tRNA-binding site or in strong-stop DNA, but the exact extent of this homology requires further investigation.

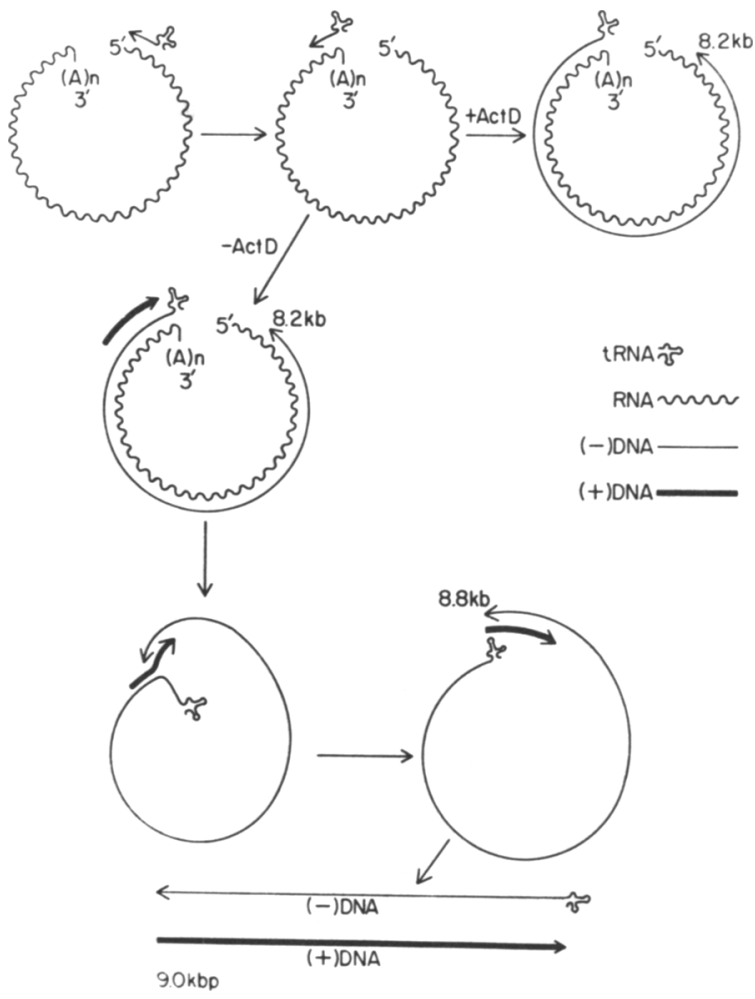

Figure 6. A model of reverse transcription. The model shows the initial production of strong-stop minus-strand DNA attached to the tRNA primer. After this DNA jumps to the 3 ' end of the RNA, a long minus-strand DNA is made that terminates just short of the $5^{\prime}$ end of the RNA genome. In the presence of Act D, minus-strand DNA synthesis terminates (8.2-kb DNA), but in the absence of the drug, a short piece of plus-strand DNA is made as a copy of the $5^{\prime}$ end of the minus-strand DNA. Synthesis of minus-strand DNA by jumping to the short plus-strand DNA and copying it. This jump positions the plus-strand DNA so that it can continue synthesis by copying the minus-strand DNA. The end result is the 9000-bp DS DNA.

Once DNA synthesis has jumped to the plus strand, we can then imagine plus-strand synthesis continuing with the initial piece of plus-strand DNA acting as primer. Elongation could then occur all the way to the other end of the minus-strand DNA. The extensive evidence, however, showing that plusstrand DNA is in fragments (see Weinberg 1977; Varmus et al. 1978) implies that there are other starting points along the minus strand for plusstrand synthesis and that ultimately the plus strand may be put together by the ligation of fragments. In any case, the second jump positions the primer for the plus strand at the exact end of the molecule and means that the ultimate product of reverse transcription can be completely double-stranded. In this way, we account for the fact that the infectious DNA molecules made in vitro show no $S_{1}$ nuclease sensitivity (Table 1), implying that they do not have any single strands at either end and that the priming mechanism for the synthesis of both strands must be able to leave flush ends at the two termini (unless, of course, there is a sequence at either end that is unnecessary for the infectivity of the molecule). 
Although this model is somewhat speculative and incomplete, we believe that its major elements are probably an appropriate representation of how reverse transcription occurs.

\section{Acknowledgments}

We are grateful to the M.I.T. Cell Culture Facility for providing the virus that made these studies possible. This work was supported by grants from the National Cancer Institute and by a contract from the Virus Cancer Program of the National Cancer Institute. D. B. is a Research Professor of the American Cancer Society. E. G. is a Dr. Chaim Weizmann postdoctoral fellow. F. Y. is a Special Fellow of the Leukemia Society of America.

\section{REFERENCES}

Bender, W., Y.-H. Chien, S. Chattopadhyay, P. K. Vogt, M. B. GARDNER, and N. DAVIDSON. 1978. High molecular weight RNAs of AKR, NZB, and wild mouse viruses and avian reticuloendotheliosis virus all have similar dimer structures. J. Virol. 25: 888 .

GianNi, A. M., D. Smotkin, and R. A. Weinberg. 1975. Murine leukemia virus: Detection of unintegrated doublestranded DNA forms of the provirus. Proc. Natl. Acad. Sci. 72:447.

HASELTINE, W. and D. BaltimoRe. 1976. In vivo replication of RNA tumor viruses. In Animal virology (ed. D. Baltimore et al.), p. 175. Academic Press, New York.

Haseltine, W. A., D. G. Kleid, A. Panet, E. Rothenberg, and D. BALTIMORE. 1976. Ordered transcription of RNA tumor virus genomes. J. Mol. Biol. 106: 109.

HIRT, B. 1967. Selective extraction of polyoma DNA from infected mouse cell cultures. J. Mol. Biol. 26:365.

KaHAN, E., F. KaHAN, and J. HURwiTZ. 1963. Specificity of action of actinomycin D. J. Biol. Chem. 238:2491.

Manly, K., D. F. Smoler, E. Bromfeld, and D. Balti-
MORE. 1971. Forms of DNA produced by virions of the RNA tumor viruses. J. Virol. 7:106.

RotHENBERG, E. and D. BALTIMORE. 1976. Synthesis of long representative DNA copies of the murine RNA tumor virus genome. $J$. Virol. 17:168.

- 1977. Increased length of DNA made by virions of murine leukemia virus at limiting magnesium ion concentration. J. Virol. 21: 168.

Rothenberg, E., D. J. Donoghue, and D. Baltimore. 1978. Analysis of a $5^{\prime}$ leader sequence on murine leukemia virus 21S RNA: Heteroduplex mapping with long reverse transcriptase products. Cell 13:435.

Rothenberg, E., D. Smotkin, D. Baltimore, and R. A. WEINBERG. 1977. In vitro synthesis of infectious DNA of murine leukemia virus. Nature 269:122.

SoUTHERN, E. 1975. Detection of specific sequences among DNA fragments separated by gel electrophoresis. J. Mol. Biol. 98:503.

TAYLOR, J. M. 1977. An analysis of the role of tRNA species as primers for the transcription into DNA of RNA tumor virus genomes. Biochim. Biophys. Acta 473:57.

Temin, H. and D. Baltimore. 1972. RNA-directed DNA synthesis and RNA tumor viruses. Adv. Virus Res. 17: 129.

Varmus, H. E., S. Heasley, H.-J. Kung, H. Oppermann, V. C. SMITH, J. M. BISHOP, and P. R. SHANK. 1978. Kinetics of synthesis, structure, and purification of avian sarcoma virus-specific DNA made in the cytoplasm of acutely infected cells. J. Mol. Biol. 120:55.

Verma, I. 1977. The reverse transcriptase. Biochim. Biophys. Acta 473:1.

Verma, I. M. and M. A. McKennetT. 1978. Genome organization of RNA tumor viruses. II. Physical maps of in vitro-synthesized Moloney murine leukemia virus doublestranded DNA by restriction endonucleases. J. Virol. 26: 630 .

WEINBERG, R. A. 1977. Structure of the intermediates leading to the integrated provirus. Biochim. Biophys. Acta 473:39.

Yoshimura, F. K., D. L. Steffen, and R. A. Weinberg. 1979. Restriction endonuclease cleavage of linear and closed circular murine leukemia viral DNAs: Discovery of a smaller circular form. In Oji International Seminaron Friend Virus and Friend Virus Disease. (In press). 